\title{
28. COMMISSION DES NEBULEUSES EXTRAGALACTIQUES
}

Président: M. E. P. Hubble, Mount Wilson Observatory, Pasadena 4, Cal., U.S.A.

Membres: MM. Baade, Carpenter, Erro, Holmberg, Humason, Lemaître, Lindblad, Lundmark, McVittie, Madwar, N. U. Mayall, Paraskevopoulos, Randers, Reynolds, Seyfert, H. Shapley, V. M. Slipher.

No report has been received.

Report of meetings

President: Dr E. P. Hubble.

Secretary: Dr C. K. Seyfert.

The Commission on Extra-Galactic Nebulae held three meetings to discuss informally the principal observational and theoretical problems of the extra-galactic nebulae. One resolution was drafted and adopted pointing out the desirability of having Dr $\mathrm{C}$. O. Lampland's valuable nebulae photographs published.

(I) Hubble described the atlas of photographs of extra-galactic nebulae he is preparing and which should be ready for distribution in about a year's time. The atlas will include photographs of about 200 representative nebulae of all types, mostly brighter than 13.o mag. selected from the Shapley-Ames Catalogue.

(2) The problem of the determination of the masses of extra-galactic nebulae was reviewed by Hubble. The three main sources of obtaining masses, from clusters of nebulae (virial theorem), spectrographic rotations, and from velocities of pairs of nebulae give masses differing by at least a factor of Io for nebulae of the same absolute magnitude. Intergalactic matter within the great clusters of nebulae cannot definitely be ruled out as a contributing factor to explain at least part of this discrepancy.

(3) Seyfert described the extremely wide planetary-like emission bands found in the nuclei of several spirals. In collaboration with Minkowski a search is now being made to determine whether or not similar objects show abnormally wide absorption lines in order to compare the stellar velocities in the nucleus with the velocities of the gas.

(4) Stebbins described the results of an investigation of photoelectric colours of faint elliptical nebulae which he and Whitford have recently obtained. A linear relation is found between international colour index and red shift for four clusters of nebulae, the most distant of which (Bootes) has a red shift corresponding to a velocity of $39,000 \mathrm{~km}$. $/ \mathrm{sec}$. $(18.2 \mathrm{mag} . \mathrm{pg})$ and a distance of about $7 \times 10^{7} \mathrm{ps}$. For the Bootes group the measured colour is $+\mathrm{I} \cdot 36 \mathrm{mag}$. or $0.52 \mathrm{mag}$. redder than nearby nebulae of similar type (elliptical). On the basis of the six-colour photometry of $\mathrm{M} 32$ one would expect that the red shift alone would make the nebulae in Bootes only 0.22 mag. redder than similar nearby systems. The resulting colour excess of 0.30 mag. for this group (and correspondingly smaller excesses for nearer groups) has had as yet no adequate explanation.

(5) A discussion initiated by McVittie and others brought out the desirability of closer co-operation between astronomers who are obtaining observations of extra-galactic nebulae and astronomers who are using these observations to construct models of the universe. In particular, the theorists desire that the observers include in their publications their original uncorrected observations together with details of their reductions to the adopted system.

(6) Shapley pointed out the fact that the Magellanic Clouds contain objects which definitely appear to be globular clusters, and that several of these objects are resolvable with the 60 -inch reflector. However, a thorough search has not revealed a single clustertype Cepheid variable that can definitely be associated with either Cloud, although such stars would be within the range of the Harvard reflector. 
(7) The methods involved in establishing the distance scale for the nebulae were reviewed and the uncertainties involved in each successive state were discussed at some length.

(8) The problem of the pronounced discrepancy between the total absolute magnitude of the globular clusters in the Milky Way, the Magellanic Clouds and M 3I, was discussed -but still remains unexplained.

(9) It was reported that Humason is now preparing a catalogue of the spectra and radial velocities of 500 extra-galactic nebulae. His spectra and those of Mayall indicate that $90 \%$ of the late type spirals show (OII) emission $(\lambda 3727)$, whereas only about $20 \%$ of the early elliptical nebulae show similar emissions.

These and related subjects were discussed at length to the profit of both observational and theoretical astronomers working in the field. 